\title{
Intersections
}

Canadian Journal of Music

Revue canadienne de musique

\section{Jean-Louis Leleu et Pascal Decroupet, éd. 2006. Pierre Boulez : Techniques d'écriture et enjeux esthétiques. Genève : Éditions Contrechamps. 326 p. ISBN 978-2-940068-23-2 (couverture souple).}

\section{Brice Tissier}

Volume 28, numéro 1, 2007

URI : https://id.erudit.org/iderudit/019297ar

DOI : https://doi.org/10.7202/019297ar

Aller au sommaire du numéro

Éditeur(s)

Canadian University Music Society / Société de musique des universités canadiennes

ISSN

1911-0146 (imprimé)

1918-512X (numérique)

Découvrir la revue

Citer ce compte rendu

Tissier, B. (2007). Compte rendu de [Jean-Louis Leleu et Pascal Decroupet, éd. 2006. Pierre Boulez : Techniques d'écriture et enjeux esthétiques. Genève :

Éditions Contrechamps. 326 p. ISBN 978-2-940068-23-2 (couverture souple).]

Intersections, 28(1), 181-187. https://doi.org/10.7202/019297ar

Tous droits réservés (C) Canadian University Music Society / Société de musique des universités canadiennes, 2007
Ce document est protégé par la loi sur le droit d'auteur. L'utilisation des services d'Érudit (y compris la reproduction) est assujettie à sa politique d'utilisation que vous pouvez consulter en ligne.

https://apropos.erudit.org/fr/usagers/politique-dutilisation/ 


\section{Book ReVIEWS / RECENSIONS}

Jean-Louis Leleu et Pascal Decroupet, éd. 2006. Pierre Boulez: Techniques d'écriture et enjeux esthétiques. Genève : Éditions Contrechamps. 326 p. ISBN 978-2-940068-23-2 (couverture souple).

Lorsque Michel Rigoni a fait paraître en 1998 son ouvrage Stockhausen ... un vaisseau lancé vers le ciel, son principal souci était moins la présentation d'un compositeur que l'étude et la transmission d'une œuvre musicale méconnue et complexe, pour laquelle les références faisaient défaut ou étaient difficiles d'accès. Le recueil Pierre Boulez: Techniques d'écriture et enjeux esthétiques, édité par Jean-Louis Leleu et Pascal Decroupet chez Contrechamps, s'inscrit dans cette perspective : présenter, par l'entremise de plusieurs travaux analytiques et musicologiques, l’euvre du compositeur français le plus imposant de la seconde moitié du $\mathrm{XX}^{\mathrm{e}}$ siècle. Car si l'on en connaît désormais assez bien le parcours, grâce à la biographie exhaustive de Dominique Jameux (malgré sa parution en 1984) ainsi que les écrits maintenant édités dans leur intégralité par Jean-Jacques Nattiez, il manquait en revanche une ouverture à l'œuvre musicale proprement dite, et celle des années 1950-60 en particulier. Ce livre, délibérément technique - enrichi d'un très grand nombre de schémas et autres tableaux - vient maintenant combler cette lacune.

L'oeuvre de Boulez est à la fois fascinante et complexe. À l'instar de son alter ego d'outre Rhin (Stockhausen), Boulez définit habituellement sa production musicale à l'image de la spirale : une œuvre en perpétuelle mutation. Il existe en effet chez Boulez une stratégie de l'inachèvement, ou plutôt du renoncement : toute œuvre consisterait à renoncer à une ou plusieurs possibilités de développement de ses matériaux originels, matériaux qui se verront alors réutilisés ultérieurement, dans d'autres contextes ou dans d'autres œuvres ${ }^{1}$. Il se révèle par conséquent difficile d'envisager l'étude d'une œuvre particulière de ce compositeur, nonobstant les quelques ouvrages spécialisés ayant réussi une approche unilatérale, comme par exemple le livre d'Olivier Meston (2001) sur Éclat. Mais pour cette raison fondamentale, une étude à la fois globale et synthétique de l’œuvre de Boulez étendue sur l'ensemble de sa carrière se révèlerait tant labyrinthique que problématique.

Concrètement, Pierre Boulez: Techniques d'écriture et enjeux esthétiques adopte la forme d'un ensemble de dix textes ciblant spécifiquement différents

1 Ainsi par exemple le Marteau sans maître, dont les matériaux constitutifs (et principalement les tableaux de multiplications sériels), issus originellement de la Deuxième Sonate pour piano et de Oubli signal lapidé, se verront réemployés pour Tombeau issu de Pli selon pli (ainsi que Don par ricochet), puis Cummings ist der Dichter. 
aspects, ainsi que plusieurs œuvres de l'auteur du Marteau sans maître. Il était de plus dans l'intention des éditeurs de se limiter à une approche résolument " génétique » de l’ouvre de Boulez. Envisagé suite au colloque de La Napoule organisé en 1998 par Jean-Louis Leleu et rassemblant un certain nombre de chercheurs qui travaillaient alors sur la musique de Boulez à la Fondation Paul Sacher, cet ouvrage nous présente l'état des travaux de plusieurs spécialistes actuels de Boulez, dans la continuité de leurs propres recherches. Ainsi par exemple Jean-Louis Leleu revient-il sur le Livre pour quatuor (" L'Écriture polyphonique dans le Livre pour quatuor de Pierre Boulez : aspects techniques et esthétiques », p. 13-38), œuvre dont il est présentement le spécialiste, et dont il avait supervisé la révision et l'enregistrement en $2000^{2}$. De son coté Pascal Decroupet - l'un des spécialistes de sa musique des années 1950-1960 - évoque Doubles, une œuvre charnière à mi-chemin entre la Troisième Sonate pour piano et Figures Doubles Prismes, dans " Moments doubles, figurés en prisme » (p. 133-158).

Tout en adoptant une succession relativement chronologique, les différents textes composant ce recueil peuvent se regrouper en trois catégories distinctes. En premier lieu, plusieurs travaux consacrés à un matériau ou à une œuvre spécifique, quelle qu’en soit l'approche. Nous avons déjà cité le Livre pour quatuor par Jean-Louis Leleu et Doubles par Pascal Decroupet. À ceux-ci sajoutent la fameuse Troisième Sonate pour piano, dont les arcanes de l'un des formants inédits sont présentés par Peter O’Hagan ("Antiphonie' : une étude du processus de composition », p. 109-132), et Éclat, dont Allen F. Edwards aborde les esquisses et la partition achevée sous l'angle de la forme et des structures ("Éclat/Multiples et le problème de la forme musicale dans les œuvres sérielles de Pierre Boulez », p. 159-176). Ensuite, plusieurs contributions envisagent des aspects plus globaux, voire même transversaux de l'œuvre de Boulez. C'est notamment le cas de Robert Piencikowski ${ }^{3}$, qui revient notamment sur le cahier Ia des Structures pour deux pianos selon une approche tripartite de la série généralisée : Boulez, Ligeti (l'analyste historique de la Structure Ia) et Xenakis («Inscriptions : Ligeti - Xenakis - Boulez», p. 95-108). Werner Strinz quant à lui envisage plusieurs œuvres inédites (Trois Essais pour percussion, Oubli signal lapidé entre autres) afin détablir, génétiquement parlant, la parenté et surtout les évolutions linguistiques et stylistiques entre Polyphonies X, Le Marteau sans maître et Cummings ist der Dichter ("Observations sur des 'objets retrouvés' dans l'œuvre de Pierre Boulez », p. 45-94).

Une troisième catégorie regroupe les deux derniers textes de l'ouvrage, consacrés à certains aspects moins connus de l'esthétique boulézienne. Le texte remarquable de Luisa Bassetto tout d'abord («Marginalia, ou l'opéra-fantôme de Pierre Boulez ", p. 255-298), nous propose un suivi des sources relatives aux projets opératiques et scéniques de Boulez. Si le projet d’opéra avec Jean Genet est de notoriété publique, on en connaît moins en revanche les raisons de l'abandon, ni par ailleurs les nombreux projets ultérieurs d'après Michaux, Rimbaud

2 Pierre Boulez, Livre pour quatuor, Quatuor Parisii, Assai 222082, 2000.

3 Conservateur du fonds Boulez à la Fondation Paul Sacher à Bâle et rédacteur du catalogue raisonné des esquisses du compositeur, Robert Piencikowski est également l'auteur de plusieurs articles importants sur les trajectoires génétiques des œuvres de Boulez (voir les références en fin de texte). 
et Artaud. Synthétisant de nombreux extraits de correspondance ainsi que plusieurs esquisses relatives à Marges, Luisa Bassetto nous permet de comprendre le parcours ainsi que la méthode de travail parfois difficile de Boulez dans les années 1960-75. La force de cet article réside dans la mise en lumière des pensées, des hésitations du compositeur au jour le jour : un projet initial dont nous pouvons voir ici la genèse, ses écueils successifs (dont la communication avec Genet), jusqu'aux refontes inachevées des idées tant littéraires (par exemple un Héliogabale d'après Artaud) que musicales (Marges donc, une œuvre conçue d'après les esquisses de ces différents projets et laissée inédite pour des raisons matérielles), avec en parallèle, l'analyse rigoureuse - toujours sources à l'appui - de fragments musicaux composés à chaque étape.

Quant au texte final, celui de Rosângela Pereira de Tugny (« Lautre moitié de l'art », p. 299-317), il aborde la dimension réellement esthétique de l'écriture boulézienne - dimension que le compositeur préfère quant à lui chroniquement éluder ${ }^{4}$ l'influence des musiques extra-européennes découvertes, entre autres, au cours des tournées entreprises à titre de directeur musical de la compagnie Renaud-Barrault, puis grâce à André Schaeffner, alors responsable du département d'ethnologie musicale au Musée de l'homme. Une influence qui transparaît particulièrement dans l'usage qu'il fera de la percussion non conventionnelle à partir du Marteau sans maître, mais aussi dans ses questionnements sur le timbre. Pour ce texte, Rosângela Pereira de Tugny choisit de décortiquer une œuvre particulière : "Constellation-Miroir », formant central de la Troisième Sonate pour piano, à la lumière de la pensée d'Artaud et des musiques extraeuropéennes.

Lautre aspect fondamental de cet ouvrage concerne sa qualité ou sa valeur plus musicologique, ce qui s'explique par un partenariat pour cette publication entre les éditeurs et la Fondation Paul Sacher de Bâle. Lorsque le célèbre mécène et chef d'orchestre suisse décide en 1986 de la création d'une fondation pour la préservation des fonds de manuscrits des compositeurs du $\mathrm{XX}^{\mathrm{e}}$ siècle, son souhait est de permettre au plus grand nombre l'accès à ces documents - trop souvent remisés dans les caves inaccessibles des bibliothèques détat - afin de favoriser la connaissance et surtout la compréhension de la genèse des grandes partitions du siècle dernier. Détenteur des fonds de Stravinski, de Bartók et de Webern par exemple, Paul Sacher, en tant que père et maître d'œuvre de la fondation qui porte son nom, persuadera également les jeunes générations de cette nécessaire préservation. Ainsi l'œuvre de Boulez sera-t-elle intégralement préservée jusqu’à la moindre esquisse ou le moindre brouillon, et consultable sur demande.

En ce qui a trait à la diffusion, la Fondation Paul Sacher développe également plusieurs projets visant à la parution des manuscrits d'œuvres phares. Après les Symphonies d'instruments à vent de Stravinski, puis la Musique pour cordes, percussions et célesta de Bartók, ce fut le tour du manuscrit de la première mise au

4 " J'ai horreur de traiter verbalement ce qu’on nomme avec complaisance le problème d'esthétique. [ ... ] Je préfére retourner à mon papier réglé » (Boulez 1995, 248). On doit par ailleurs à Rosângela Pereira de Tugny la publication de la correspondance entre Pierre Boulez et André Schaeffner (publiée chez Fayard en 1998). 
net du Marteau sans maître (Boulez 1952-54). Le présent ouvrage s'inscrit aussi dans la continuité de cette démarche : la propagation et la compréhension des arcanes, de la substantifique moelle des œuvres de Boulez. Grâce à un cd-rom joint à l'ouvrage, le lecteur pourra consulter et posséder les fac-similés d'un très grand nombre d'esquisses, numérisées et en couleur, en lien avec les différents articles présentés au sein du livre. Ces esquisses, pour la plupart inédites, reflètent par ailleurs un aspect fondamental du processus créateur chez Boulez : le compositeur-théoricien pensant ses œuvres sur de longues périodes, développant, par des schémas et croquis généralement esquissés sur du papier à lettre à l'entête d'hôtels américains ou allemands ${ }^{5}$, ce qui deviendra un jour - par exemple - le plan général de la Troisième Sonate pour piano (p. 109-115), les tableaux d'engendrement des hauteurs avec les graphies, nomenclatures et couleurs choisies par Boulez, ou encore les différentes manières de redéployer ces tableaux dans telle ou telle future composition (p. 45-94).

Si ce livre est particulièrement innovant et remarquable sur les plans technique et musicologique, et cela parce qu'il permet au lecteur une intrusion dans le travail créateur de Boulez en l'analysant et en dévoilant du matériel de première main, nous pourrions cependant lui adresser un reproche substantiel : celui du choix des œuvres abordées. En soi, il est tout à fait compréhensible de voir les spécialistes de cette musique lappréhender selon des angles personnels et souvent pointus, mais l'analyse de pièces plus accessibles aurait peut-être été plus pédagogique pour le lecteur non familier avec ces inédits. Pour donner un exemple clair : la Troisième Sonate pour piano est facilement accessible au disque et en partition en ce qui concerne ses deux formants édités, mais il n’en est rien des autres formants, dont il est régulièrement question dans le cadre de cet ouvrage. Même constat pour Doubles : le travail remarquable de Pascal Decroupet sur cette première version de Figures Doubles Prismes ne saurait être illustré par aucun enregistrement ni aucune partition. Il n'est par ailleurs pas possible de se procurer la partition de la version terminale de l'œuvre, restée - et probablement définitivement - à l'état de work in progress. De la même manière, on pourrait regretter l'absence d'études d'œuvres postérieures à 1975, passant ainsi sous silence les dernières trente années de la production boulézienne. Un reproche qui sapplique aussi à la biographie de Jameux (1984) n’excédant pas le Répons de 1984.

Mais on soulignera tout de même deux textes notables consacrés à deux œuvres majeures et facilement accessibles : Le Marteau sans maitre et Rituel in memoriam Bruno Maderna, respectivement par Pascal Decroupet et Jean-Louis Leleu, et Paolo dal Molin. CEuvre probablement la plus étudiée du compositeur, Le Marteau sans maître avait fait l'objet, en 1990, d'un travail particulièrement poussé de la part de Lev Koblyakov (1990) dans A world of harmony. Koblyakov y détaillait notamment le travail d'élaboration des hauteurs, les techniques bouléziennes de multiplication de complexes sonores, ainsi que leurs déploiements au sein de la partition. Mais on avait déploré à lépoque certains manques quant au réel travail de composition, c'est-à-dire de réalisations rythmique et struc-

5 Ou encore sur du papier millimétré. Cf. la note $n^{\circ} 4$. 
turelle de ces travaux préparatoires ${ }^{6}$. Le texte " 'Penser sensiblement' la musique : production et description du matériau harmonique dans le troisième mouvement du Marteau sans maître " (p. 177-215) vient combler cette lacune. À travers l'étude de la seule troisième pièce du cycle (pour flûte en sol et voix), les auteurs nous proposent un véritable décryptage multi-angulaire de la genèse de ces quelques cinquante mesures, à la lumière encore une fois des esquisses préparatoires, et des tableaux d'engendrement des rythmes issus d'Oubli signal lapidé. Un travail précis et abouti parce qu'il nous permet de voir clairement et dans le détail un certain nombre de processus d'engendrement au niveau cellulaire en mettant pour la première fois en rapport deux œuvres jusquà présent dissociées. Il en va de même pour Rituel in memoriam Bruno Maderna, probablement l'une des œuvres les plus rigoureuses de son auteur - mais aussi l'une des plus accessibles - tant par sa forme et ses structures que par son langage. Il était déjà connu que cette œuvre était à lorigine une marge de la matrice d' ... explosante-fixe ... de 1972, dont elle reprenait les encadrés (soit sept gestes musicaux fondés sur les sept notes de la série originelle) ${ }^{7}$. Paolo Dal Molin nous propose dans son texte (" ... 'sans cause extérieure apparente, ni affluents, ni glaciers, ni orages' ... La construction de l'hétérophonie dans les versets de Rituel », p. 217-254) un parcours détaillé de toutes les étapes intermédiaires, de toutes les réflexions successives entre cet état originel et le développement ultime, donc de la partition finale telle que nous pouvons l'entendre désormais. Ces deux contributions fourniront au lecteur soucieux d'aborder, et surtout de comprendre l'œuvre de Boulez, deux approches claires et efficaces du compositeur.

À l'issue de ces considérations, nous pouvons affirmer que les dix écrits qui composent cet ouvrage permettront sans aucun doute de franchir un pas non négligeable quant à l'approche musicologique et historique de l’œuvre de Boulez, au risque de rendre peut-être obsolète un grand nombre de travaux analytiques antérieurs qui, en l'absence de références concrètes aux esquisses, ne pouvaient espérer expliquer certains aspects du langage du compositeur. Ce qui ne saurait enlever en rien, bien évidemment, leur valeur méthodologique et musicale et l'apport scientifique qu'ils ont portés au moment de leur publication ${ }^{8}$.

6 Koblyakov lui-même n'aborde que très peu la question du rythme dans le premier cycle du Marteau. De même, il ne fait quévoquer subrepticement dans une note de bas de page, et sans détail aucun, la parenté pourtant fondamentale avec Oubli signal lapidé (Koblyakov 1990, 32).

7 Nous renvoyons pour cela à la matrice proprement dite, publiée en mémoire de Stravinski dans la revue Tempo en 1972, et reprise dans la pochette du disque d' ... explosante-fixe ... , Sophie Cherrier (flûte Midi solo), Emmanuelle Ophèle et Pierre-André Valade (flûtes), Ensemble InterContemporain, Andrew Gerszso (IRCAM/réalisation électroacoustique), dir. Pierre Boulez, Deutsche Grammophon DG 445 833-2, 1995.

8 Pour donner un autre exemple : Célestin Deliège avait proposé en 1977 une approche analytique de la Première Improvisation sur Mallarmé, où il traitait principalement les questions du rapport textemusique, ainsi que les convergences paradigmatiques des différentes sections de la partie vocale. Dans une note de bas de page, il indique toutefois renoncer à la question de la genèse des structures, notamment en raison de la difficulté de "la soumettre à une hypothèse unique » (Deliège 1986, 149). Depuis, nous savons grâce aux esquisses de la Fondation Paul Sacher que la partition vocale constitue la refonte d'une section de Strophes (1957) pour flûte seule, entrecoupée de deux des Notations pour piano retranscrites pour l'ensemble instrumental. 
Alors que de très nombreux ouvrages ont paru depuis des années sur l'œuvre boulézienne en langue anglaise ou allemande ${ }^{9}$, on était en droit de réclamer, justement, un ouvrage technique dans la langue même de Boulez, mais aussi du René Char du Visage Nuptial et du Marteau sans maître, du Mallarmé de Pli selon pli et du Michaux de Poésie pour pouvoir! Signalons que cette perspective est actuellement au cœur des préoccupations de la maison dédition Contrechamps, qui publie par ailleurs plusieurs traductions d'ouvrages en langue française, notamment les ouvrages d'Adorno et de Dahlhaus ainsi que les écrits de Huber ou de Bartók. Autre détail important quant à ce choix d’éditeur : Contrechamps et son directeur Philippe Albèra (un autre spécialiste de l’œuvre du compositeur) ont édité en 2003 un ouvrage consacré à Boulez, et plus particulièrement à son cycle Pli selon pli d’après Mallarmé.

Pour conclure, Pierre Boulez: Techniques d'écriture et enjeux esthétiques restera un livre principalement conçu par des spécialistes, et destiné a priori pour des spécialistes ou du moins pour des mélomanes éclairés. Il ne saurait constituer en soi un outil didactique de première approche de l'œuvre de Boulez. Mais il a le mérite de tenter d'extraire cette Euvre majeure du XXe siècle du cénacle d'initiés dans lequel elle était traditionnellement circonscrite, et cela afin de la rendre accessible, toute réserve technique gardée, au plus grand nombre. En somme, ce livre fera référence en ce qui concerne létude musicologique de l'œuvre musicale de Pierre Boulez.

\section{RÉFÉRENCES}

Albèra, Philippe, éd. 2003. Pli selon pli de Pierre Boulez : Entretiens et études. Genève : Éditions Contrechamps.

Boulez, Pierre. 1972. "Canons and Epitaphs set 2", Tempo 98.

1995. Points de repère. Tome I Imaginer, nouvelle édition entièrement refondue. Textes réunis par Jean-Jacques Nattiez et Sophie Galaise. Paris : Christian Bourgois Éditeur.

2005. 1952-54. Le Marteau sans maître : Fac-simile de lépure et de la première mise au net de la partition, éd. par Pascal Decroupet. Londres/ Mainz : Schott.

Boulez, Pierre, et André Schaeffner. 1998. Correspondance 1954-1970, présentée et annotée par Rosângela Pereira de Tugny. Paris : Fayard.

Deliège, Célestin. 1986. “The Convergence of two poetic systems". Dans Pierre Boulez: a symposium, éd. William Glock, 99-126. Londres: Eulenbourg Books. Traduit en français la même année dans Célestin Deliège, Invention musicale et idéologies, 139-70. Paris : Christian Bourgois Éditeur.

Glock, William, ed. 1986. Pierre Boulez: a symposium. Londres : Eulenbourg Books.

Jameux, Dominique. 1984. Pierre Boulez. Paris : Fayard.

Koblyakov, Lev. 1990. A world of harmony. New York : Harwood Academic Publishers.

9 Par exemple, l'ouvrage dirigé par William Glock (1986) abordant notamment l’ensemble des œuvres de jeunesse à la lumière des manuscrits inédits. 
Meston, Olivier. 2001. Éclat de Pierre Boulez. Paris : Éditions Michel de Maule. Piencikowski, Robert. 1983. «'Assez lent, suspendu, comme imprévisible' : quelques aperçus sur les travaux d'approche d'Éclat ». Genesis 4 : 51-67. 1985. "Nature morte avec guitare ». Dans Pierre Boulez: eine Festschrift zum 60. Geburtstag am 26. März 1985, éd. par J. Häusler, 66-81. Vienne: Universal Editions.

Rigoni, Michel. 1998. Stockhausen ... un vaisseau lancé vers le ciel. Paris : Millénaire III Éditions.

\section{Discographie}

Boulez, Pierre. 2000. Livre pour quatuor. Quatuor Parisii. Assai 222082.

Boulez, Pierre. 1995. ... explosante-fixe. ... Sophie Cherrier (flûte Midi solo), Emmanuelle Ophèle et Pierre-André Valade (flûtes), Ensemble InterContemporain, Andrew Gerszso (IRCAM/réalisation électroacoustique), dir. Pierre Boulez. Deutsche Grammophon DG 445 833-2.

Brice Tissier

George et Ira Gershwin. 2000. Pardon My English: Vocal Score. Édité par Steven D. Bowen. Paroles et musique de George et Ira Gershwin. Livret de Herbert Fields et Morrie Ryskind. Miami, Floride : Warner Bros Music Corp. xxiii, 357 p. ISBN 0-7692-9201-1, ISBN 978-0-7692-9201-4 (couverture rigide).

Avec cette partition de Pardon My English, le chef dorchestre et orchestrateur Steven D. Bowen propose ici la première édition critique d'une comédie musicale de George et Ira Gershwin largement méconnue, si on la compare avec l'opéra Porgy and Bess. Cette publication s'inscrit dans le cadre du Leonore S. Gershwin - Library of Congress Recording and Publishing Project, une initiative lancée en 1989 par Leonore, la veuve du librettiste. À partir des archives de Warner et de la Library of Congress, le projet a pour objectif la restauration de quatre comédies musicales des frères Gershwin : Lady, Be Good!, Strike Up the Band, Girl Crazy et Pardon My English (Dalrymple et Bowen 1994). Une première étape a visé l'enregistrement de chacune des œuvres. La suite (présentement en cours) prévoit que chaque comédie musicale fera l'objet d'une édition critique de la partition chant et piano. La première réalisation de léquipe remonte à 1998, avec l'édition de Strike Up the Band, dirigée par Steven D. Bowen.

Si les œuvres des Jerome Kern, Irving Berlin, Cole Porter, George Gershwin et Richard Rodgers contribuent fortement à l'essor de la comédie musicale américaine des années 1920, la crise économique de 1929 vient quelque peu freiner la progression du genre. Alors qu'une dizaine de productions de Broadway connaissent plus de 500 représentations dans les années 1920, seulement trois spectacles dépassent le cap des 400 représentations dans les années 1930 : Anything Goes et DuBarry Was a Lady de Porter, ainsi qu'Of Thee I Sing de Gershwin (Block 2002, 78). En 1927-1928, plus de 50 nouvelles productions 